\title{
A Survey of the Experiences and Perceptions of Midwifery Nurses on the Mom Connect Programme in Bojanala, South Africa
}

\section{SB Pitse* and H Van Der Heever}

Department of Health Sciences, Sefako Makgatho Health Sciences University, South Africa

*Corresponding author: Pitse SB, Department of Health Sciences, Sefako Makgatho Health Sciences University, South Africa, E-mail: bonolo.pitse@yahoo.com

Received date: August 13, 2018; Accepted date: August 21, 2018; Published date: August 24, 2018

Copyright: $@ 2018$ Pitse SB, et al. This is an open-access article distributed under the terms of the Creative Commons Attribution License, which permits unrestricted use, distribution, and reproduction in any medium, provided the original author and source are credited.

\begin{abstract}
MomConnect is a form of mobile health technology which requires healthcare workers to register and confirm the client's pregnancy on a national database using a cell phone. This programme is driven by primary users such as midwifery nurses, hence the need to obtain their views. The objectives of this study were to determine the experiences and perceptions of midwifery nurses regarding the MomConnect programme in Bojanala health district, North West Province in South Africa. A quantitative, cross-sectional survey was done with 100 midwifery nurses offering antenatal care in Rustenburg sub-district primary healthcare facilities. Mixed perceptions and experiences were identified. The majority of the respondents found MomConnect to be an acceptable way to provide continuous care $(97 \%, n=97)$ and further support its continued use $(87 \%, n=87)$. On the other hand, some respondents viewed MomConnect as extra work $(52 \%, n=52)$, impossible to integrate into routine care $(39 \%, n=39)$ and disrupting the workflow at times $(44 \%, n=44)$.
\end{abstract}

Keywords: Mobile health (mHealth); MomConnect; Midwifery nurses; Maternal and child care; Antenatal

\section{Introduction}

The World Health Organisation (WHO) reported that 99\% of maternal-related deaths occur in developing countries, which includes South Africa, and that most of these deaths are preventable. Furthermore, one of the recommendations resulting from the Commission on Information and Accountability (CoIA) for women's and children's health established by the UN in 2011, is integration of information and communication technology in healthcare services. There is a need for multi-communication and capacity building for women and their families in order to promote safer pregnancies, for example increasing awareness by educating clients on danger signs of pregnancy [1].

In the effort to promote safer pregnancy and possibly reduce maternal and child mortality rates, Peter et al. [2] observe that a form of mobile health technology which requires healthcare workers to register and confirm the client's pregnancy on a national database using a cell phone, were initiated by some countries. Through the short message system, the pregnant women will then receive pregnancy stage-specific messages, messages about good nutrition, danger signs in pregnancy, HIV and other messages that promote healthy living as well as messages about the infant post-delivery, for one year. In South Africa, this form of mobile health as launched by the National Department of Health in 2014 is known as MomConnect.

Not much has been explored and documented on the acceptance levels, experiences and perceptions of healthcare workers before and throughout the implementation of this programme. This is a challenge because failure to explore perceptions and experiences of healthcare workers, who are mainly the primary entry points, may impact negatively on the success, sustainability and expansion of the programme, as evidenced by studies conducted on mobile health [3].
Consequently, the potential of this programme to capacitate women and reduce maternal and child mortality rates will not be realised if frontline healthcare workers do not implement it. Any health programme will stand a better chance of successful implementation and improvement if the voices of primary implementers such as nurses are heard. MomConnect depends on healthcare workers-even if women can self-register, they still need to go to the health facility for full registration and receipt of a full set of text messages.

Aranda-Jan et al. [4] also recommend a follow-up of projects or programmes in order to adapt designs in a way that suits the context and therefore increase chances of them being successful. This study is therefore necessary as it is concerned with active involvement of healthcare workers, who are vital to the success of the MomConnect programme in delivering health-related messages to the registered pregnant women.

\section{Methodology}

\section{Study design and study setting}

A quantitative, cross-sectional survey was done. MomConnect is mainly used during basic antenatal care, which is the core function of primary healthcare facilities. The study was therefore conducted in primary healthcare facilities of the Rustenburg health sub-district, which fall under the Rustenburg local municipality in Bojanala Platinum District Municipality in the North West Province, South Africa. There were twenty primary healthcare facilities in Rustenburg sub-district during the time of this study, all offering antenatal care.

\section{Study population and sample}

The study population was all midwifery nurses working in Rustenburg sub-district primary healthcare facilities and who have provided antenatal care after the launch of the MomConnect programme in 2014. The number of midwifery nurses in the twenty 
Citation: Pitse SB, Van Der Heever H (2018) A Survey of the Experiences and Perceptions of Midwifery Nurses on the Mom Connect Programme in Bojanala, South Africa. Clinics Mother Child Health 15: 298. doi:10.4172/2090-7214.1000298

Page 2 of 5

clinics was found to be 144, but only 100 midwifery nurses had offered antenatal care and used MomConnect. Using 100 midwifery nurses as the study population, a $95 \%$ confidence interval and a $5 \%$ margin of error, the sample size would be 80 [5]. Therefore, due to the scarcity and hence the small size of the study population, all 100 nurses were included in the study to ensure that study objectives would be met as well as to minimise sampling error.

\section{Data collection and analysis}

A structured, self-administered questionnaire was used to collect data relevant to the study's objectives from the midwifery nurses working in the twenty primary healthcare facilities in Rustenburg health sub-district.

The raw data were captured, cleaned and coded in Microsoft Excel (Microsoft Office 2013) and also verified for correctness. After capturing raw data into Microsoft Excel spreadsheet, STATA 13 (Small Stata 13.0) software was used to import and analyse data. The results were described in frequency distribution for demographics and summarised by means of descriptive summary measures expressed as mean, median and range for numerical or continuous variables and percentages for categorical variables.

The Fisher exact test was carried out to determine whether perceptions, experiences and socio-demographic aspects (e.g. age, gender) indicate a relationship with the use of the MomConnect programme.

\section{Ethical considerations}

The ethical clearance certificate was granted by the Sefako Makgatho Health Sciences University Ethical Committee, with reference number SMUREC/H/215/2016: PG.
Permission to conduct the study was further granted by the North West Province Health Policy, Planning and Research Department; Bojanala health district; Kgetleng health sub-district (for pre-testing the questionnaire) and Rustenburg health sub-district, where the study was conducted. Respondents were fully informed of the aims and objectives of the study, given an information leaflet, and those willing to participate in the study signed the consent form.

\section{Results}

\section{Demographic characteristics}

The respondents comprised midwifery nurses who offer antenatal care and use the MomConnect programme in the Rustenburg health sub-district, Bojanala district in South Africa $(n=100)$. The majority of respondents were females, $92 \%(n=92)$, fell into the age category of $46-55$ years $(38 \%, n=38)$, possessed a diploma in nursing as the highest qualification $(85 \%, n=85)$, were permanently employed $(90 \%, n=90)$, and had more than 10 years' work experience $(62 \%, n=62)$.

\section{Experiences of respondents regarding MomConnect utilisation}

The experiences of midwifery nurses regarding the use of MomConnect are summarised in Table 1.

The mean time it takes to register pregnant women on MomConnect is approximately $9 \mathrm{~min}$. Of the 100 respondents, it respectively takes $5 \%(n=5)$ less than 5 min to register; $80 \%(n=80)$ 5-10 min to register and $15 \%(n=15)$ more than $10 \mathrm{~min}$ to register women on MomConnect.

\begin{tabular}{|c|c|c|}
\hline Variable & Frequency $(n=100)$ & Percent (\%) \\
\hline \multicolumn{3}{|c|}{ It is easy to register women on MomConnect } \\
\hline Agree & 88 & 88 \\
\hline Not sure & 11 & 11 \\
\hline No & 1 & 1 \\
\hline Total & 100 & 100 \\
\hline \multicolumn{3}{|c|}{ I have registered all pregnant women that I offered antenatal care to on MomConnect } \\
\hline Agree & 16 & 16 \\
\hline Not sure & 12 & 12 \\
\hline Disagree & 72 & 72 \\
\hline Total & 100 & 100 \\
\hline \multicolumn{3}{|c|}{ It is possible to integrate MomConnect registration into routine antenatal care } \\
\hline Agree & 43 & 43 \\
\hline Not sure & 18 & 18 \\
\hline Disagree & 39 & 39 \\
\hline Total & 100 & 100 \\
\hline
\end{tabular}


Citation: Pitse SB, Van Der Heever H (2018) A Survey of the Experiences and Perceptions of Midwifery Nurses on the Mom Connect Programme in Bojanala, South Africa. Clinics Mother Child Health 15: 298. doi:10.4172/2090-7214.1000298

Page 3 of 5

\begin{tabular}{|c|c|c|}
\hline \multicolumn{3}{|c|}{ Do women registered on MomConnect ever come back to the health facility to ask for clarity about messages they have received? } \\
\hline Yes & 28 & 28 \\
\hline Not sure & 14 & 14 \\
\hline No & 58 & 58 \\
\hline Total & 100 & 100 \\
\hline \multicolumn{3}{|c|}{ MomConnect programme facilitates communication between the women and nurses } \\
\hline Agree & 89 & 89 \\
\hline Not sure & 8 & 8 \\
\hline Disagree & 3 & 3 \\
\hline Total & 100 & 100 \\
\hline \multicolumn{3}{|c|}{ Registering women on MomConnect can disrupt the workflow } \\
\hline Agree & 44 & 44 \\
\hline Not sure & 10 & 10 \\
\hline Disagree & 46 & 46 \\
\hline Total & 100 & 100 \\
\hline \multicolumn{3}{|c|}{ I support continued use of MomConnect } \\
\hline Agree & 87 & 87 \\
\hline Not sure & 12 & 12 \\
\hline Disagree & 1 & 1 \\
\hline Total & 100 & 100 \\
\hline
\end{tabular}

Table 1: Experiences of midwifery nurses at Rustenburg sub-district $(\mathrm{n}=100)$.

In terms of reasons of MomConnect non-registration, $45 \%(\mathrm{n}=45)$ mentioned clients not having a cell phone as a challenge; $42 \%(n=42)$ mentioned that other staff categories such as counsellors assisted with registrations; $26 \%(\mathrm{n}=26)$ had challenges with clients not having identity documents (IDs); $15 \%(\mathrm{n}=15)$ mentioned poor network conditions while trying to register clients; $12 \%(\mathrm{n}=12)$ reported not having enough time as a barrier; $4 \%(n=4)$ reported language barriers in terms of clients from other countries who do not understand languages that are used for MomConnect messages; and 2\% $(n=2)$ could not register women because their facility-specific MomConnect code did not work.

\section{Perceptions of respondents regarding MomConnect programme}

The perceptions of midwifery nurses regarding the MomConnect programme are summarised in Table 2.

\section{Possible associations were detected between these variables}

Perceived usefulness of MomConnect programme and its continued use ( $\mathrm{p}=0.008$, Fisher exact test);

Perceived usefulness of MomConnect programme and its acceptability ( $\mathrm{p}=0.031$, Fisher exact test);
MomConnect registration viewed as extra work and its integration into routine antenatal care $(\mathrm{p}=0.015$, Fisher exact test $)$; and

MomConnect registration experienced as disrupting the workflow and its integration into routine antenatal care $(\mathrm{p}=0.000$, Fisher exact test).

\begin{tabular}{|c|c|c|}
\hline Variable & Frequency $(n=100)$ & Percent (\%) \\
\hline \multicolumn{3}{|c|}{$\begin{array}{l}\text { MomConnect is an acceptable way of providing continuous healthcare to } \\
\text { pregnant women }\end{array}$} \\
\hline Agree & 97 & 97 \\
\hline Not sure & 2 & 2 \\
\hline Disagree & 1 & 1 \\
\hline Total & 100 & 100 \\
\hline \multicolumn{3}{|c|}{$\begin{array}{l}\text { MomConnect has the potential to improve the quality of maternal and child } \\
\text { care }\end{array}$} \\
\hline Agree & 96 & 96 \\
\hline Not sure & 4 & 4 \\
\hline Disagree & 0 & 0 \\
\hline
\end{tabular}




\begin{tabular}{|c|c|c|}
\hline Total & 100 & 100 \\
\hline \multicolumn{3}{|c|}{$\begin{array}{l}\text { MomConnect is useful in empowering pregnant women on self-care anc } \\
\text { child care }\end{array}$} \\
\hline Agree & 97 & 97 \\
\hline Not sure & 3 & 3 \\
\hline Disagree & 0 & 0 \\
\hline Total & 100 & 100 \\
\hline
\end{tabular}

Women registered on MomConnect will refrain from consulting nontrusted sources for information

\begin{tabular}{|l|l|l|}
\hline Agree & 49 & 49 \\
\hline Not sure & 35 & 35 \\
\hline Disagree & 16 & 16 \\
\hline Total & 100 & 100 \\
\hline
\end{tabular}

MomConnect programme is the nurses' support system that complements face-to-face maternal care given to women by nurses

\begin{tabular}{|l|l|l|}
\hline Agree & 88 & 88 \\
\hline Not sure & 9 & 9 \\
\hline Disagree & 3 & 3 \\
\hline Total & 100 & 100 \\
\hline
\end{tabular}

Registering women on MomConnect is extra work

\begin{tabular}{|l|l|l|}
\hline Agree & 52 & 52 \\
\hline Not sure & 7 & 7 \\
\hline Disagree & 41 & 41 \\
\hline Total & 100 & 100 \\
\hline
\end{tabular}

There is a chance that women registered on MomConnect will misinterpret the messages sent to them

\begin{tabular}{|l|l|l|}
\hline Agree & 41 & 41 \\
\hline Not sure & 38 & 38 \\
\hline Disagree & 21 & 21 \\
\hline Total & 100 & 100 \\
\hline
\end{tabular}

Registration on MomConnect breaks confidentiality of the women's information

\begin{tabular}{|l|l|l|}
\hline Agree & 6 & 6 \\
\hline Not sure & 7 & 7 \\
\hline Disagree & 87 & 87 \\
\hline Total & 100 & 100 \\
\hline
\end{tabular}

Table 2: MomConnect perceptions of midwifery nurses at Rustenburg sub-district $(\mathrm{n}=100)$.

\section{Discussion}

This study, similar to other mHealth studies, highlights the conflicting experiences and perceptions regarding mHealth programmes. Generally, respondents support the continued use of MomConnect, view it as an easy and acceptable way of facilitating communication, empowering women and hence having the potential to improve maternal and child health. At the same time, some respondents see MomConnect as extra work, disruptive of the workflow and difficult to integrate into routine antenatal care.

A struggle to integrate mHealth into routine healthcare was identified as well in some studies, with health workers being more likely to see mHealth as running parallel to other services [6-8]. In a contrasting finding, Van Zutphen et al. [9] found that midwives in Netherlands could integrate mHealth into their routine services. This contrast may be due to a well-developed health system and adequate allocation of midwifery nurses who care for antenatal and postnatal women in Netherlands, as opposed to the increased workload versus the limited number of midwifery nurses that are available in some primary healthcare facilities in South Africa.

Moreover, integration of MomConnect programme into routine antenatal care is negatively affected when midwifery nurses experience MomConnect as extra work and as disruptive to the workflow. However, Farmani et al. [10] remark that even if people have a negative attitude towards a particular technology, they will use it if they believe that it is useful. This is possibly why midwifery nurses support the continued use of MomConnect programme even if they view it as being extra work and disruptive.

In terms of issues of confidentiality, the majority of respondents thought that MomConnect registration does not breach any confidentiality concerning the women's information. In contrast, health workers in a study by Gagnon et al. [11] were concerned about issues of confidentiality and they felt that they did not have control over messages that women receive. These contrasting findings may possibly be because this study focused on text messaging only, which respondents may view as less intrusive, while the systematic review by Gagnon et al. [11] also included other mobile technologies such as the mobile electronic medical record system, smartphone, tablet, etc., which may be viewed as less confidential.

Age, gender, level of education and years of experience were less significant in terms of the experiences and perceptions of midwifery nurses on the MomConnect programme, which is in contrast with other studies. This is possibly because all respondents in this study had tertiary qualifications and viewed Mom Connect as useful and acceptable. mHealth programmes that are perceived to be useful and easy to use, are more easily accepted and adopted by users $[8,11-14]$.

\section{Limitations}

The sample size was small and this could impact negatively on generalisation of the findings. However, the researcher conducted a census of the study population to promote representativeness. Some respondents stated that the MomConnect programme can be disruptive to the workflow and is impossible to integrate into their routine, but due to the nature of the study design, it was not possible to further explore the ways in which this disruption occurred, or what factors limit the integration. 
Citation: Pitse SB, Van Der Heever H (2018) A Survey of the Experiences and Perceptions of Midwifery Nurses on the Mom Connect Programme in Bojanala, South Africa. Clinics Mother Child Health 15: 298. doi:10.4172/2090-7214.1000298

Page 5 of 5

\section{Conclusion}

Midwifery nurses generally view MomConnect as an acceptable and potential way of improving the quality of care, and also support its continued use, but there are mixed and sometimes conflicting perceptions and experiences, in line with the findings of other mHealth studies. This study also emphasised that there is interplay between user perceptions and experiences or utilisation of mHealth.

Reasons for non-registration of women on MomConnect were highlighted, and the misconception that ID numbers are a necessity for registration was picked up. The implications are that health promotion messages do not reach some women, and this impacts negatively on the aim of MomConnect of improving the quality of maternal and child care through continuous communication.

\section{Recommendations}

Follow-up of projects or programmes must be conducted by relevant staff, such as mother-and-child healthcare coordinators, in order to gather information on good practices and challenges, and to clear any misconceptions. This study revealed that some women were not registered on MomConnect because they did not have IDs, but women can still be registered with their date of birth, and not with ID numbers only. Although there is an increased workload in primary healthcare facilities, all midwifery nurses must at least be familiar with MomConnect so that they can interact with women where necessary.

In addition, MomConnect registrations can be reviewed monthly or quarterly during facility and sub-district performance reviews, so that its value can consistently be emphasised. Respondents viewed these text messages as having the potential to improve the quality of maternal and child care through empowering pregnant women from pregnancy up until at one year after birth. Although the MomConnect costs were not evaluated, these messages may be useful if expanded to other priority programmes like tuberculosis, whereby both nurses and clients can receive educational and supportive messages, and possibly improve TB outcomes such as TB treatment success rate and reduce lost to follow-up rate.

In terms of research, more usability studies that will include other categories of staff, as well as clients that use mHealth, are needed in order to get the bigger picture of experiences and perceptions of frontline users on mHealth. Since some midwifery nurses were not familiar with MomConnect, it may be valuable to also study their awareness and knowledge of NurseConnect (an extension of MomConnect in which supportive messages are sent to nurses).

\section{Acknowledgements}

The authors would like to acknowledge: the Policy, Planning, Research, Monitoring and Evaluation department of the North West health Province, Bojanala health district and Rustenburg health subdistrict for agreeing to the study being conducted. A special thank you to all the participating midwifery nurses for their cooperation and valuable responses.

\section{References}

1. Coleman A (2014) The Use of ICT Tools (Mobile Phones) to improve awareness of Pregnancy Danger Signs among Pregnant Women in Rural Communities of South Africa. J Commun 5: 203-209.

2. Peter J, Barron P, Pillay Y (2016) Using mobile technology to improve maternal, child and youth health and treatment of HIV patients. S Afr Med J 106: 3-4.

3. Brinkel J, Kramer A, Krumkamp R, May J, Fobil J (2014) Mobile phonebased mHealth approaches for public health surveillance in Sub-Saharan Africa: a systematic review. Int J Environ Res Public Health 11: 11559-11582.

4. Aranda-Jan CB, Mohutsiwa-Dibe N, Loukanova S (2014) Systematic review on what works, what does not work and why of implementation of mobile health (mHealth) projects in Africa. BMC Public Health 14: 1.

5. Raosoft Inc (2004) Sample size calculator.

6. Leon N, Schneider H, Daviaud E (2012) Applying a Framework for Assessing the Health System Challenges to Scaling up mHealth in South Africa. BMC Medical Informatics and Decision Making 12: 123.

7. Labrique AB, Vasudevan L, Kochi E, Fabricant R, Mehl G (2013) mHealth innovations as health system strengthening tools: 12 common applications and a visual framework. Glob Health Sci Prac 1: 160-171.

8. Willcox JC, van der Pligt P, Ball K, Wilkinson SA, Lappas M (2015) Views of Women and Health Professionals on mHealth Lifestyle Interventions in Pregnancy: A Qualitative Investigation. JMIR Mhealth Uhealth 3: e99.

9. Van Zutphen M, Milder IE, Bemelmans WJ (2009) Integrating an eHealth Program for Pregnant Women in Midwifery Care: A Feasibility Study among Midwives and Program Users. J Med Internet Res 11: e7.

10. Farmani M, Kimiaee A, Fatollahzadeh F (2012) Investigation of Relationship between ease of use, innovation tendency, perceived usefulness and intention to use technology: An empirical study. Indian J Sci Technol 5: 3678-3682.

11. Gagnon MP, Ngangue P, Payne-Gagnon J, Desmartis M (2016) M-Health adoption by healthcare professionals: a systematic review. J Am Med Inform Assoc 23: 212-220.

12. Davis FD (1989) Perceived Usefulness, Perceived Ease of Use, and User Acceptance of Information Technology. MIS Quart 13: 319-340.

13. Park Y, Chen JV (2007) Acceptance and adoption of the innovative use of smartphone. Indust Manag Data Syst 107: 1349-1365.

14. Kaphle S, Chaturvedi S, Chaudhuri I, Krishnan R, Lesh N (2015) Adoption and Usage of mHealth Technology on Quality and Experience of Care Provided by Frontline Workers: Observations from Rural India. JMIR Mhealth Uhealth 3: e61. 